\title{
Rational use and effectiveness of morphine in the palliative care of cancer patients at the Ocean Road Cancer Institute in Dar es salaam, Tanzania
}

\author{
A. KAMUHABWA* and D. EZEKIEL \\ Unit of Pharmacology and Therapeutics, School of Pharmacy, Muhimbili University of Health and Allied \\ Sciences, P.O. Box 65013, Dar es Salaam Tanzania
}

\begin{abstract}
Morphine and other opioids is the mainstay of cancer pain management. However, considerable fears surrounding their use present barriers to pain control. The aim of this study was to assess the rational use and effectiveness of morphine for management of pain in the palliative care of cancer patients at Ocean Road Cancer Institute (ORCI) in Tanzania. A total of 100 cancer patients who were receiving morphine therapy at the ORCI were interviewed to get information on morphine use. In addition, information on the prescribed doses of morphine was obtained from medical records of 200 patients who have used morphine from September 2005 to April 2006. Both outpatients and inpatients with advanced cancer who were receiving morphine for palliative care were involved. Seven (7) palliative caregivers, including two doctors, two nurses, a pharmacist, a pharmaceutical technician and a social worker were also interviewed. Of the 100 interviewees, $37 \%$ were aware of morphine. The level of education and duration of therapy had an impact on the awareness. The results also showed that oral morphine solution was the most common route (96\%) of administration. Fifty-seven percent of the patients described the doses of morphine given to be effective in relieving their pain. Although most patients $(79 \%)$ experienced morphine-induced side effects, the majority (93\%) were continuing with the therapy. There were no indication of irrational use of morphine and morphine-induced side effects were well managed. The majority of patients and caregivers had positive attitude towards the use of morphine. In conclusion, the study revealed that the use of morphine is acceptable among a large proportion of patients receiving palliative care and that the majority of them find the doses given effective to relieve their pain.
\end{abstract}

Key words: cancer, patient, pain control, morphine, palliative care, hospital, Tanzania

\section{Introduction}

Cancer is now becoming an important public problem in developing countries. The disease is characterized by pain which is suffered by $50-70 \%$ of the patients and it becomes severe as the disease progresses (WHO, 1990; Angarola, 1990; Powis et al., 2004). For this reason control of pain and of psychological, social and spiritual problems is very important so as to provide the best quality of life for patients and their families. Palliative care is an approach that improves the quality of life of patients and their families facing the problem associated with life threatening illnesses by the prevention and relief of suffering. It is an approach to pain management therefore careful assessment of pain is essential. Palliative care is achieved by means of early identification, impeccable assessment and treatment of pain and other symptoms be it physical, psychosocial and spiritual (WHO, 1990).

In the management of pain, World Health Organization (WHO, 1996) guidelines advocate the use of analgesics "by the mouth, by the clock and by the analgesic ladder". This involves control of pain by pharmacological means grouped into three categories, namely: control of mild pain using non-steroidal anti-inflammatory drugs (NSAIDS) like paracetamol, diclofenac, aspirin and others; control of moderate pain by using weak opioids like codeine and tramadol; and control of severe pain using strong opioids like morphine and pethidine (Cleeland, 1993; WHO, 1996).

In developing countries pain and palliative care are poorly understood and are traditionally given low priority in national health care systems (WHO, 1996). There is lack of education for health care professionals, policy makers and the public that exaggerates fears of the effects of morphinelike drugs thus limiting their use by patients and health care workers alike. Obstacles to effective cancer pain relief worldwide also include poor drug availability, misguided national drug legislation, under-prescribing and under-dosing by the professionals and wrong timing of drugs 
given and lack of public awareness that pain can be controlled. In addition, some anti-drug abuse laws and regulations restrict their medical availability and use (WHO, 1996).

Morphine may be prescribed to patients at all stages of cancer. In reality, the patients most likely to experience pain, and likely also to have the most severe pain, are those with metastatic disease. These patients may yet have many months to live, but their quality of life is adversely affected by pain, since unrelieved pain leads to social isolation, loss of role and depressed mood. Treatment success is influenced by many factors like proper dosing and maximum adherence to therapy. Poor adherence can be due to poor perception to the drug. This is especially important in palliative care where use of morphine and other opioids is associated with beliefs that these drugs are given as last resort for dying patients (Powis et al., 2004). It is therefore important to assess the rational use of morphine in palliative care of cancer patients for the purpose of ensuring proper pain control. To our knowledge, no study has been conducted in Tanzania to address the perception of care personnel and patients on the use of morphine for palliative care as well as the doses of morphine solutions used in treating different pain conditions in cancer patients. The aim of this study was therefore to assess the perception of patients and caregivers towards the use of morphine at the Ocean Road Cancer Institute in Tanzania.

\section{Materials and Methods}

\section{Study area}

The study was conducted at Ocean Road Cancer Institute (ORCI) in Dar es Salaam, Tanzania. ORCI is the only referral hospital for cancer cases in Tanzania. At ORCI patients are managed by chemotherapy, radiotherapy and other means including palliative care. The institute attends to nearly 2500 new cancer patients per year. Among these, 80\% have advanced incurable diseases. Moreover, there are about 3000 patients coming for follow up, of which nearly $60 \%$ have disease recurrence and progression. This makes the number of patients who need palliative care to be around 3800 per year (ORCI Reports, unpubl.).

\section{Data collection}

The study consisted of 100 cancer patients with chronic pain and who were receiving morphine therapy at the ORCI between October 2006 and
April 2007. These patients were interviewed in order to get the information on morphine use. A questionnaire with open and close-ended questions was used to collect information from patients. The questionnaire was structured in English and translated in Kiswahili for easy communication with patients. Both outpatients and inpatients with advanced cancer that were symptomatic and receiving morphine for palliative care were involved in the study. The selection of patients included men and women of different age groups ( $\geq 14$ years old). The patients were conveniently selected based on the state of their illnesses and the ability to freely give the information.

Medical records of 200 patients(including 100 patients who were interviewed in this study and another 100 whose information was obtained from patient's records) were reviewed to gather information on the doses, duration and formulations of morphine that were given to patients. In addition, the types of cancers among patients who were receiving morphine therapy were recorded from patient's files. The files included in the study were randomly selected and included those of patients who have been using morphine from September 2005 to April 2006. A form was designed and used to record patient information from their files.

Seven palliative caregivers, including two doctors, two nurses, a pharmacist, a pharmaceutical technician and a social worker were interviewed. Self-administered English questionnaires were used to gather information. Open and closed-ended questions were used to get information on the perception of morphine use in palliative care, criteria that are used to assess the need to increase doses of morphine to patients, incidences of morphine induced tolerance, side effects and their management.

\section{Ethical considerations}

Before being interviewed, patients were given a description of the study and asked if they were willing to participate in the study. Verbal consent was sufficient for one to be included in the study. Permission to conduct the study was granted by the ORCI management.

\section{Data analysis}

Data was analysed by using EPI-Info 2002 computer software programme. Descriptive analysis was undertaken and chi-square test was used to test significant differences in proportions between the different variables in the study. 


\section{Results}

Of the 100 patients interviewed, $44.0 \%$ and $56.0 \%$ were males and females respectively. The majority $(40 \%)$ of the interviewee was $\geq 51$ years old. About 36\% (20/56) of females were aware that morphine was used to control severe pain. On the other hand, a slightly smaller proportion $30 \%(13 / 44)$ of males was aware that morphine was the drug of choice for controlling severe pain in cancer patients. Majority of the patient (63\%) mentioned that they had never heard of morphine but confirmed using it when asked if they are given a drug in a liquid form to relieve pain. For those who responded that they were aware of the morphine they were asked to mention the uses of the drug. Majority of them $(78 \%)$ indicated that it is used to relieve pain while $28 \%$ said it is used for the purpose of sedation.

Patients were asked about the severity of pain they were experiencing before given morphine. The results showed that $95 \%$ had very severe pain, $5 \%$ had severe pain and none of them had mild or moderate pain when was given the drug. When asked the duration of time one has been using morphine, $52.0 \%$ of the patients indicated to have used morphine for less than 30 days, $41.0 \%$ used it for 1-6 months while only $7 \%$ have used it for more than 6 months.

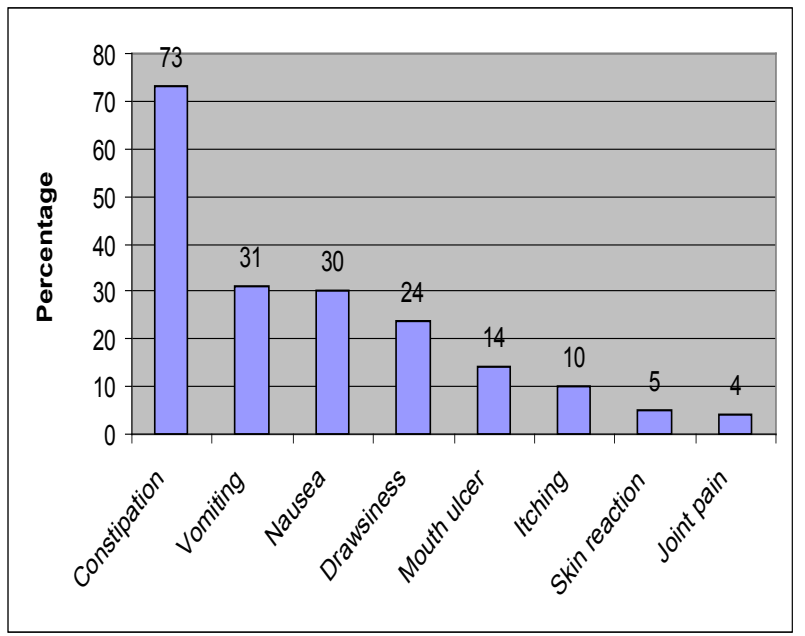

Figure 1: Common morphine-induced side effects reported by the patients $(N=100)$

Majority of respondents $(57 \%)$ indicated that the doses they received were adequate to relieve pains. Thirty two percent indicated that the effect of the doses was moderate, whereas a small proportion (11\%) indicated that the doses given did not relieve the pain. Patients $(43 \%)$ who felt the initial doses given did not adequately relieve their pain were asked on the measures that were taken by their care providers in order to relieve their pains. Majority $(78 \%)$ of them mentioned that the doses of morphine were increased, others $(13 \%)$ were given another drug and in very few cases $(9 \%)$ no further measures were taken. Seventy-nine percent of the patients reported to experience side effects, but $94 \%$ of them reported that they continued to use morphine despite the side effects. Constipation was the commonest side effect which was reported (32.9\%) followed by vomiting and nausea (Figure 1).

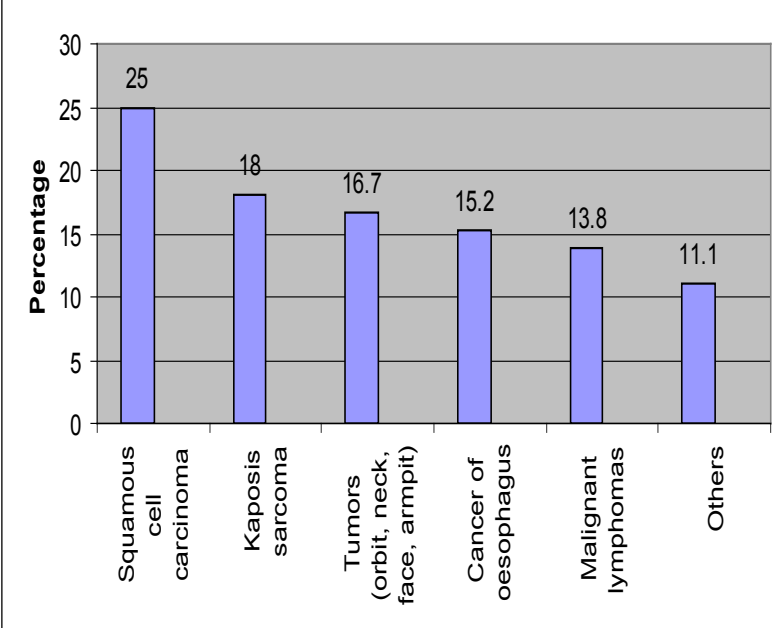

Figure 2: Common types of cancers among male patients receiving morphine therapy

Among 200 patients whose records were studied males were 72 while females were 128 . Squamous cell carcinoma was the commonest type of cancer among males accounting for 25\% followed by Kaposi's sarcoma (18\%) (Figure 2). Cervical cancer was the leading type of cancer (35\%) followed by breast cancer $(23.4 \%)$ among the female patients (Figure 3).

Seven palliative care workers were also interviewed. The caregivers responded that morphine was prescribed to patients with severe pain which is not responding to NSAIDS and weak opioids. The doses of morphine that were prescribed for the management of pain ranged from $5 \mathrm{mg} / \mathrm{mls}$ to $50 \mathrm{mg} / 5 \mathrm{mls}$. These doses were titrated according to severity of pain and adjusted according to response. All the caregivers indicated that the use of morphine was safe, acceptable and effective for relieving pain associated with chronic cancer. Most frequently mentioned side effects were constipation, drowsiness, headache, nausea and vomiting all of which are manageable. 


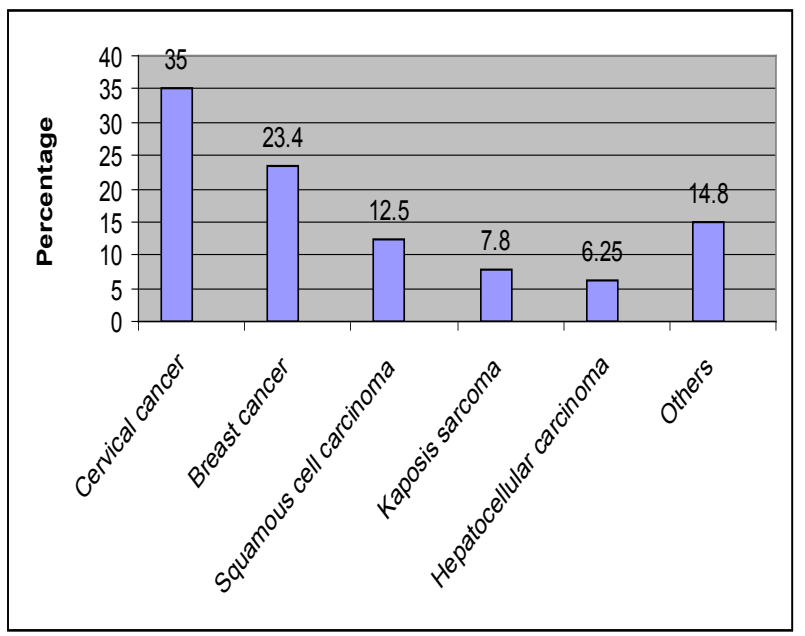

Figure 3: Common types of cancers among female patients receiving morphine therapy

\section{Discussion}

Like in many other studies in Tanzania, the results of this study indicate that cervical cancer is the leading type of cancer for females followed by breast cancer $(23.4 \%$ ) (Ngwalle et al., 2001; Kidanto et al., 2002; Mayaud et al., 2003). On the other hand, the commonest type of cancer in males was squamous cell carcinoma followed by Kaposi's sarcoma (18\%).

For many years now morphine has been the most successful opioid in alleviating pain in cancer patients. Despite its usefulness as analgesic the exaggerated fears of its effects limits its use by patients and health care workers. Opiophobia phenomenon results in morphine under use and existence of some myths concerning morphine use. These include the fear that morphine is dangerous because it causes respiratory depression and it is also addictive (Thompson et al., 1995). For some patients being prescribed with morphine, means that they are going to die soon hence fear of death surrounds them (Powis et al., 2004). Some medical practitioners regard morphine as the last drug to prescribe to patients and they believe that it should be reserved when death is imminent (Cleeland, 1993).

The results of the study revealed that onethird $(33 \%)$ of the respondents were aware of the drugs they were using for pain relief. Oral route was the most common route of administration. This complies with WHO guidelines which state that in palliative care analgesics should be given 'by mouth' unless oral route is not suitable to the patient (WHO, 1990). This is to minimize pain that could be caused by frequent injections.
In this study only $4 \%$ of the patients were given morphine by injection because of several reasons including, vomiting and difficult in swallowing because of painful ulceration in the throat.

In our study, just over half of the patients reported that the doses of morphine were effective to relieve their pain. Similar findings have been reported elsewhere (Portenoy \& Foley, 1996). Doses of morphine that are given to patients at ORCI range from $5 \mathrm{mg} / \mathrm{ml}$ to $50 \mathrm{mg} / 5 \mathrm{ml} 4$ hourly. A study conducted in Israel showed that only $12 \%$ of the patients needed more than $300 \mathrm{mg}$ per day (i.e. $50 \mathrm{mg} 4$ hourly) (Bercovitch et al., 1999). Taken together, all these findings show compliance with WHO recommendations (WHO, 1990).

The results of the present study have also shown that one-third of the patients needed their doses to be increased and over three quarters of these patients had their doses increased in the course of treatment. The results also indicated that morphine is well tolerated. These findings are supported by reports from other studies elsewhere which showed that only a small proportion of patients under morphine therapy stop the drug due to poor response or other reasons (Bercovitch et al., 1999; Mercadante et al., 2006). In this study the caregivers had positive attitude towards the use of morphine and that they followed analgesic ladder recommended by WHO when prescribing and administering morphine to patients (WHO, 1990).

The findings of our study further show that majority of patients experience morphineinduced side effects, the common ones being constipation and vomiting. Despite experiencing such side effects, most of these patients reported to continue using the drug, an indication that these side effects are tolerable and manageable among the majority of the patients. Other side effects such as oral ulceration and skin reaction could not be linked to the use of morphine. These are likely to be associated with chemotherapy and radiotherapy, respectively (Volpato et al., 2007).

In conclusion, the use of morphine is acceptable among a large proportion of patients receiving palliative care and that the majority of them find the doses given effective to relieve their pain. However, the level of knowledge about morphine is low in most patients. There is therefore a need to encourage patient participation to strengthen the whole process of pain management and that information, education and communication on the use of 
morphine need to be improved. It is important that more studies should are conducted to a large population of cancer patients to evaluate morphine use and its effectiveness in the palliative care of cancer patients in the country.

Received 11 May 2009

Revised 29 July 2009

Accepted 7 September 2009

\section{References}

Angarola, R.T. (1990) National and international regulation of opioid drugs: Purpose, structures, benefits and risks. Journal of Pain and Symptom Management 5, 6-11.

Bercovitch, M., Waller, A. \& Adunsky, A. (1999) High dose morphine use in the hospice setting. A database survey of patient characteristics and effect on life expectancy. Israel Journal of Pain 5, 871877.

Cleeland, C.S. (1993) Strategies for improving cancer pain management. Journal of Pain and Symptoms Management 8, 361-365.

Kidanto, H.L., Kilewo, C.D. \& Moshiro, C. (2002) Cancer of the cervix: knowledge and attitudes of female patients admitted at Muhimbili National Hospital, Dar es Salaam. East African Medical Journal 79, 467-475.

Mayaud, P., Weiss H.A., Lacey, C.J., Gill, D.K. \& Mabey, D.C. (2003) Genital human papillomavirus genotypes in northwestern Tanzania. Journal of Clinical Microbiology 41, 4451-4453.

Mercadante, S., Porzio, G., Ferrera, P. \& Fulfaro, F. (2006) Low morphine doses in opioid naive cancer patients with pain. Italian Journal of Pain Symptoms Management 3, 242-247.

Ngwalle, E.W., Mgaya, H.N., MpanjuShumbusho, W., Chirenje, Z.M., Kirumbi, L., Lebelle, T. \& Kaggwa, S. (2001) Situational analysis for diagnosis and treatment of cervical cancer in mainland Tanzania. East African Medical Journal 78, 60-64.

Portenoy, R.K. \& Foley, K.M. (1996) Chronic use of opioid analgesics in cancer pain: Report of 38 cases. Pain 25, 171-186.

Powis, J., Etchells, E., Martin D., MacRae, S. \& Singer, P. (2004) Can a "good death" be made better?: A preliminary evaluation of a patient-centred quality improvement strategy for severely ill in-patients. BMC Palliative Care 3, 2-8.

Thompson, P.I., Joel, S.P., John, L., Wedzicha, J,A,, Maclean, M. \& Slevin, M.L. (1995) Respiratory depression following morphine and morphine-6-glucuronide in normal subjects. British Journal of Clinical Pharmacology 40, 145-152.

Volpato, L.E., Silva, T.C., Oliveira, T.M., Sakai, V.T. \& Machado, M.A. (2007) Radiation therapy and chemotherapy-induced oral mucositis. Brazilian Journal of Otorhinolaryngology 73, 562-568.

WHO (1990) Cancer Relief and Palliative Care. WHO Expert Committee. Geneva, World Health Organization.

WHO (1996) Cancer Pain Relief, with a Guide to opioid availability. $2^{\text {nd }}$ ed. Geneva: World Health Organization. 